Preprint of the paper

"A Boundary Element Numerical Approach for Earthing Grid Computation" I. Colominas, F. Navarrina, M. Casteleiro (1999)

Computer Methods in Applied Mechanics \& Engrng., 174 , 73-90.

http://caminos.udc.es/gmni 


\title{
A Boundary Element Numerical Approach for Grounding Grid Computation
}

\author{
I. Colominas, F. Navarrina, M. Casteleiro
}

Dpto. de Métodos Matemáticos y de Representación, E.T.S. de Ingenieros de Caminos, Canales y Puertos; Universidad de La Coruña. Campus de Elviña, 15192 La Coruña, SPAIN

\begin{abstract}
Analysis and design of substation earthing involves computing the equivalent resistance of grounding systems, as well as distribution of potentials on the earth surface due to fault currents $[1,2]$. While very crude approximations were available in the sixties, several methods have been proposed in the last two decades, most of them on the basis of intuitive ideas such as superposition of punctual current sources and error averaging [3,4]. Although these techniques represented a significant improvement in the area of earthing analysis, a number of problems have been reported; namely: large computational requirements, unrealistic results when segmentation of conductors is increased, and uncertainty in the margin of error [4].

A Boundary Element approach for the numerical computation of substation grounding systems is presented in this paper. Several widespread intuitive methods (such as the Average Potential Method) can be identified in this general formulation as the result of suitable assumptions introduced in the BEM formulation to reduce computational cost for specific choices of the test and trial functions. On the other hand, this general approach allows the use of linear and parabolic leakage current elements to increase accuracy. Efforts have been particularly made in getting a drastic reduction in computing time by means of new completely analytical integration techniques, while semi-iterative methods have proven to be specially efficient for solving the involved system of linear equations. This BEM formulation has been implemented in a specific Computer Aided Design system for grounding analysis developed within the last years. The feasibility of this approach is finally demonstrated by means of its application to two real problems.
\end{abstract}




\section{Introduction}

\subsection{Mathematical Model of the Physical Problem}

A safe earthing system has to guarantee the integrity of equipment and the continuity of the service under fault conditions - providing means to carry and dissipate electrical currents into the ground - and to safeguard that persons working or walking in the surroundings of the grounded installation are not exposed to dangerous electrical shocks. To achieve these goals, the equivalent electrical resistance of the system must be low enough to assure that fault currents dissipate mainly through the grounding grid into the earth, while maximum potential differences between close points on the earth surface must be kept under certain tolerances (step, touch and mesh voltages) [1,2].

Physical phenomena underlying fault currents dissipation into the earth can be modelled by means of Maxwell's Electromagnetic Theory [5]. Constraining the analysis to electrokinetic steady-state response $[1,6]$, and neglecting the inner resistivity of the earthing electrode (a system of interconnected buried conductors), the 3D problem associated with an electrical current derivation to earth can be written as

$$
\begin{gathered}
\operatorname{div}(\boldsymbol{\sigma})=0, \quad \boldsymbol{\sigma}=-\boldsymbol{\gamma} \operatorname{grad}(V) \text { in } E \\
V=V_{\Gamma} \text { in } \Gamma, \quad \boldsymbol{n}_{E} \cdot \boldsymbol{\sigma}=0 \text { in } \Gamma_{E} .
\end{gathered}
$$

where $E$ is the earth, $\boldsymbol{\gamma}$ its conductivity tensor, $\Gamma_{E}$ the earth surface, $\boldsymbol{n}_{E}$ its normal exterior unit field and $\Gamma$ the electrode surface $[7,8]$. The solution to this problem gives the potential $V$ and the current density $\boldsymbol{\sigma}$ at an arbitrary point $x$ when the electrode attains a voltage $V_{\Gamma}$ (Ground Potential Rise or GPR) relative to a distant grounding point assumed to be at the potential of remote earth.

In these terms, being $\boldsymbol{n}$ the normal exterior unit field to $\Gamma$, the leakage current density $\sigma$ at an arbitrary point of the earthing electrode surface, the ground current $I_{\Gamma}$ (total surge current being leaked into the earth) and the equivalent resistance of the earthing system $R_{e q}$ (apparent resistance of the electrodeearth circuit) can be written as

$$
\sigma=\boldsymbol{\sigma}^{t} \boldsymbol{n}, \quad I_{\Gamma}=\iint_{\Gamma} \sigma d \Gamma, \quad R_{e q}=\frac{V_{\Gamma}}{I_{\Gamma}}
$$

For most practical purposes, the assumption of homogeneous and isotropic soil can be considered accurate [2], and the tensor $\boldsymbol{\gamma}$ can be substituted by a measured apparent scalar conductivity $\gamma$. Otherwise, a multi-layer model 
could be accepted without risking a serious calculation error [2]. Since the kind of techniques described in this paper can be extended to multi-layer soil models $[9,20]$ (representing the ground as stratified into two or more layers of appropriate thickness, each one with a different value of $\gamma$ ), further discussion and examples are restricted to uniform soil models. Hence, problem (1) reduces to the Laplace equation with mixed boundary conditions [5].

On the other hand, if one further assumes that the earth surface is horizontal, symmetry (method of images) allows us to rewrite (1) in terms of the Dirichlet Exterior Problem:

$$
\begin{array}{r}
\Delta V=0 \text { in } E \\
V=V_{\Gamma} \text { in } \Gamma \text { and } \Gamma^{\prime}
\end{array}
$$

where the image surface $\Gamma^{\prime}$ is the symmetric of $\Gamma$ with respect to the earth surface $[7,8]$. The assumption of horizontal earth surface seems to be quite adequate, if we consider that surroundings of almost every electrical installation must be leveled before its construction. Further assumption $V_{\Gamma}=1$ is not restrictive at all, since $V$ and $\boldsymbol{\sigma}$ are proportional to $V_{\Gamma}$.

\section{Variational Statement of the Problem}

In most electrical installations, the earthing electrode consists of a grid of interconnected bare cylindrical conductors, horizontally buried and supplemented by a number of vertical rods, which ratio diameter/length is relatively small $\left(\sim 10^{-3}\right)$. Obviously, no analytical solutions can be obtained for this kind of problem. Moreover, this specific geometry precludes the use of standard numerical techniques (such as Finite Differences or Finite Elements [11]) since discretization of domain $E$ is required, and obtaining sufficiently accurate results should imply unacceptable computing efforts in memory storage and cpu time.

However, computation of potential is only required on the earth surface and the equivalent resistance can be easily obtained (2) in terms of the current density $\sigma$ that leaks from the electrode surface. Thus, we turn our attention to a Boundary Integral approach, which would only require discretization of the earthing grid surface $\Gamma$, and will therefore reduce the three-dimensional problem to a two-dimensional one.

Application of Green's Identity [12,13] to (1) allows us to obtain the following expression for the potential $V$ in $E$, in terms of the unknown leakage current 
density $\sigma$ :

$$
V(\boldsymbol{x})=\frac{1}{4 \pi \gamma} \iint_{\boldsymbol{\xi} \in \Gamma} k(\boldsymbol{x}, \boldsymbol{\xi}) \sigma(\boldsymbol{\xi}) d \Gamma
$$

with the weakly singular kernel

$$
k(\boldsymbol{x}, \boldsymbol{\xi})=\left(\frac{1}{r(\boldsymbol{x}, \boldsymbol{\xi})}+\frac{1}{r\left(\boldsymbol{x}, \boldsymbol{\xi}^{\prime}\right)}\right), \quad r(\boldsymbol{x}, \boldsymbol{\xi})=|\boldsymbol{x}-\boldsymbol{\xi}|
$$

where $\boldsymbol{\xi}^{\prime}$ is the symmetric of $\boldsymbol{\xi}$ with respect to the earth surface $[7,8]$.

Since (4) holds [8] on the earthing electrode surface $\Gamma$ and the potential is known by the boundary condition on the GPR $(V(\chi)=1, \chi \in \Gamma)$, the leakage current density $\sigma$ must satisfy the Fredholm integral equation of the first kind defined on $\Gamma$

$$
1=\frac{1}{4 \pi \gamma} \iint_{\boldsymbol{\xi} \in \Gamma} k(\boldsymbol{\chi}, \boldsymbol{\xi}) \sigma(\boldsymbol{\xi}) d \Gamma, \quad \chi \in \Gamma
$$

Finally, a weaker variational form [10] of equation (6) can now be written as:

$$
\iint_{\boldsymbol{x} \in \Gamma} w(\boldsymbol{\chi})\left[1-\frac{1}{4 \pi \gamma} \iint_{\boldsymbol{\xi} \in \Gamma} k(\boldsymbol{\chi}, \boldsymbol{\xi}) \sigma(\boldsymbol{\xi}) d \Gamma\right] d \Gamma=0
$$

which must hold for all members $w(\boldsymbol{\chi})$ of a suitable class of test fuctions defined on $\Gamma$.

Obviously, a Boundary Element approach seems to be the right choice to solve variational statement $(7)$.

\subsection{Boundary Element Formulation}

For a given set of $\mathcal{N}$ trial functions $\left\{N_{i}(\boldsymbol{\xi})\right\}$ defined on $\Gamma$, and for a given set of $\mathcal{M} 2 \mathrm{D}$ boundary elements $\left\{\Gamma^{\alpha}\right\}$, the unknown leakage current density $\sigma$ and the earthing electrode surface $\Gamma$ can be discretized in the form

$$
\sigma(\boldsymbol{\xi})=\sum_{i=1}^{\mathcal{N}} \sigma_{i} N_{i}(\boldsymbol{\xi}), \quad \Gamma=\bigcup_{\alpha=1}^{\mathcal{M}} \Gamma^{\alpha}
$$


and expression (4) can be approximated as

$$
\begin{aligned}
& V(\boldsymbol{x})=\sum_{i=1}^{\mathcal{N}} \sigma_{i} V_{i}(\boldsymbol{x}), \quad V_{i}(\boldsymbol{x})=\sum_{\alpha=1}^{\mathcal{M}} V_{i}^{\alpha}(\boldsymbol{x}), \\
& V_{i}^{\alpha}(\boldsymbol{x})=\frac{1}{4 \pi \gamma} \iint_{\boldsymbol{\xi} \in \Gamma^{\alpha}} k(\boldsymbol{x}, \boldsymbol{\xi}) N_{i}(\boldsymbol{\xi}) d \Gamma^{\alpha} .
\end{aligned}
$$

Moreover, for a given set of $\mathcal{N}$ test functions $\left\{w_{j}(\chi)\right\}$ defined on $\Gamma$, the variational statement ( 7 ) is reduced to the system of linear equations

$$
\begin{aligned}
& \sum_{i=1}^{\mathcal{N}} R_{j i} \sigma_{i}=\nu_{j}, \quad j=1, \ldots, \mathcal{N} ; \\
& R_{j i}=\sum_{\beta=1}^{\mathcal{M}} \sum_{\alpha=1}^{\mathcal{M}} R_{j i}^{\beta \alpha}, \quad \nu_{j}=\sum_{\beta=1}^{\mathcal{M}} \nu_{j}^{\beta} ; \\
& R_{j i}^{\beta \alpha}=\frac{1}{4 \pi \gamma} \iint_{\boldsymbol{\chi} \in \Gamma^{\beta}} w_{j}(\boldsymbol{\chi}) \iint_{\boldsymbol{\xi} \in \Gamma^{\alpha}} k(\boldsymbol{\chi}, \boldsymbol{\xi}) N_{i}(\boldsymbol{\xi}) d \Gamma^{\alpha} d \Gamma^{\beta} \\
& \nu_{j}^{\beta}=\iint_{\boldsymbol{\chi} \in \Gamma^{\beta}} w_{j}(\boldsymbol{\chi}) d \Gamma^{\beta} .
\end{aligned}
$$

In practice, the $2 \mathrm{D}$ discretization required to solve the above stated equations in real problems implies an extremely large number of degrees of freedom. In addition if we take into account that the coefficients matrix in (11) is full and the computation of each contribution (13) requires double integration on 2D domains, we conclude that some additional simplifications must be introduced to overcome the extremely high computational cost of the problem.

\section{Approximated 1D Variational Statement}

With this scope, and considering the characteristic geometry of grounding grids in most of real electrical installations, one can assume that the leakage current density is constant around the cross section of the cylindrical electrode $[7,8]$. This hypothesis of circumferential uniformity is widely used in most of the theoretical developments and practical techniques related in the literature $[1,2,4]$.

Let $L$ be the whole set of axial lines of the buried conductors, $\hat{\boldsymbol{\xi}}$ the orthogonal projection over the bar axis of a given generic point $\boldsymbol{\xi} \in \Gamma, \phi(\hat{\boldsymbol{\xi}})$ the electrode diameter, $C(\widehat{\boldsymbol{\xi}})$ the circumferential perimeter of the cross section at $\widehat{\boldsymbol{\xi}}$, and 
$\hat{\sigma}(\hat{\boldsymbol{\xi}})$ the approximated leakage current density at this point (assumed uniform around the cross section). Thus, expression (4) can be written in the form

$$
\hat{V}(\boldsymbol{x})=\frac{1}{4 \pi \gamma} \int_{\hat{\boldsymbol{\xi}} \in L}\left[\int_{\boldsymbol{\xi} \in C(\widehat{\boldsymbol{\xi}})} k(\boldsymbol{x}, \boldsymbol{\xi}) d C\right] \hat{\sigma}(\widehat{\boldsymbol{\xi}}) d L
$$

Now, since the leakage current is not exactly uniform around the cross section, boundary condition $V(\boldsymbol{\chi})=V_{\Gamma}=1, \boldsymbol{\chi} \in \Gamma$ will not be strictly satisfied at every point $\chi$ on the electrode surface $\Gamma$, and variational equality (7) will not hold anymore. However, if we restrict the class of trial functions to those with circumferential uniformity, that is $w(\chi)=\widehat{w}(\hat{\boldsymbol{\chi}}) \forall \chi \in C(\hat{\chi}),(7)$ results in:

$$
\int_{\hat{\boldsymbol{\chi}} \in L} \hat{w}(\hat{\boldsymbol{\chi}})\left[\pi \phi(\hat{\boldsymbol{\chi}})-\frac{1}{4 \pi \gamma} \int_{\hat{\boldsymbol{\xi}} \in L} K(\hat{\boldsymbol{\chi}}, \hat{\boldsymbol{\xi}}) \hat{\sigma}(\hat{\boldsymbol{\xi}}) d L\right] d L=0
$$

which must hold for all members $\widehat{w}(\hat{\chi})$ of a suitable class of test fuctions defined on $L$, being the integral kernel

$$
K(\hat{\chi}, \widehat{\boldsymbol{\xi}})=\int_{\boldsymbol{\chi} \in C(\hat{\chi})}\left[\int_{\boldsymbol{\xi} \in C(\widehat{\boldsymbol{\xi}})} k(\boldsymbol{\chi}, \boldsymbol{\xi}) d C\right] d C
$$

In this way, the boundary condition has to be satisfied on the average at every cross section. In fact, (16) can be considered as a weaker variational statement of the Fredholm integral equation of the first kind on $L$

$$
\pi \phi(\hat{\boldsymbol{\chi}})=\frac{1}{4 \pi \gamma} \int_{\hat{\boldsymbol{\xi}} \in L} K(\hat{\boldsymbol{\chi}}, \hat{\boldsymbol{\xi}}) \hat{\sigma}(\hat{\boldsymbol{\xi}}) d L \quad \forall \hat{\boldsymbol{\chi}} \in L
$$

Since ends and junctions of conductors are not taken into account in this formulation, slightly anomalous local effects are expected at these points, although global results should not be noticeably affected in real problems.

\subsection{D Boundary Element Formulation}

Resolution of integral equation (16) involves discretization of the domain formed by the whole set of axial lines of the buried conductors $L$. Thus, for given sets of $n$ trial functions $\left\{\widehat{N}_{i}(\widehat{\boldsymbol{\xi}})\right\}$ defined on $L$, and $m 1 \mathrm{D}$ boundary elements $\left\{L^{\alpha}\right\}$, the unknown approximated leakage current density $\hat{\sigma}$ and the set of axial lines $L$ can be discretized in the form

$$
\hat{\sigma}(\hat{\boldsymbol{\xi}})=\sum_{i=1}^{n} \hat{\sigma}_{i} \widehat{N}_{i}(\hat{\boldsymbol{\xi}}), \quad L=\bigcup_{\alpha=1}^{m} L^{\alpha}
$$


and a discretized version of the approximated potential (15) can be obtained as

$$
\begin{aligned}
& \hat{V}(\boldsymbol{x})=\sum_{i=1}^{n} \hat{\sigma}_{i} \hat{V}_{i}(\boldsymbol{x}), \quad \hat{V}_{i}(\boldsymbol{x})=\sum_{\alpha=1}^{m} \hat{V}_{i}^{\alpha}(\boldsymbol{x}), \\
& \hat{V}_{i}^{\alpha}(\boldsymbol{x})=\frac{1}{4 \pi \gamma} \int_{\hat{\boldsymbol{\xi}} \in L^{\alpha}}\left[\int_{\boldsymbol{\xi} \in C(\hat{\boldsymbol{\xi}})} k(\boldsymbol{x}, \boldsymbol{\xi}) d C\right] \widehat{N}_{i}(\hat{\boldsymbol{\xi}}) d L .
\end{aligned}
$$

Finally, for a suitable selection of $n$ test functions $\left\{\hat{w}_{j}(\hat{\chi})\right\}$ defined on $L$, equation (16) is reduced to the system of linear equations

$$
\begin{aligned}
& \sum_{i=1}^{n} \widehat{R}_{j i} \hat{\sigma}_{i}=\hat{\nu}_{j}, \quad j=1, \ldots, n \\
& \hat{R}_{j i}=\sum_{\beta=1}^{m} \sum_{\alpha=1}^{m} \hat{R}_{j i}^{\beta \alpha}, \quad \hat{\nu}_{j}=\sum_{\beta=1}^{m} \widehat{\nu}_{j}^{\beta} ; \\
& \hat{R}_{j i}^{\beta \alpha}=\frac{1}{4 \pi \gamma} \int_{\widehat{\boldsymbol{\chi}} \in L^{\beta}} \widehat{w}_{j}(\hat{\boldsymbol{\chi}})\left[\int_{\hat{\boldsymbol{\xi}} \in L^{\alpha}} K(\hat{\boldsymbol{\chi}}, \widehat{\boldsymbol{\xi}}) \widehat{N}_{i}(\widehat{\boldsymbol{\xi}}) d L\right] d L, \\
& \hat{\nu}_{j}^{\beta}=\int_{\widehat{\boldsymbol{\chi}} \in L^{\beta}} \pi \phi(\hat{\boldsymbol{\chi}}) \widehat{w}_{j}(\hat{\boldsymbol{\chi}}) d L .
\end{aligned}
$$

On a regular basis, the computational work required to solve a real problem is drastically reduced by means of this $1 \mathrm{D}$ formulation with respect to the 2D formulation given in section $\mathbf{2}$, mainly because the size of the linear equations system (22) and the number of contributions (24) that is necessary to calculate are expected to be significantly smaller than those in (11) and (13).

However, extensive computing is still required for integration. Since integrals on the circumferential perimeter of electrodes are taken separate from integrals on their axial lines, we look forward to reducing the high computational effort required for circumferential integration in (17) and (21). Obviously, further simplifications are necessary to reduce computing time under acceptable levels $[8]$.

\subsection{Simplified 1D Boundary Element Formulation}

The inner integral of kernel $k(\boldsymbol{x}, \boldsymbol{\xi})$ in $(21)$ can be written as:

$$
\int_{\boldsymbol{\xi} \in C(\widehat{\boldsymbol{\xi}})} k(\boldsymbol{x}, \boldsymbol{\xi}) d C=\int_{\boldsymbol{\xi} \in C(\widehat{\boldsymbol{\xi}})} \frac{1}{r(\boldsymbol{x}, \boldsymbol{\xi})} d C+\int_{\boldsymbol{\xi} \in C(\widehat{\boldsymbol{\xi}})} \frac{1}{r\left(\boldsymbol{x}, \boldsymbol{\xi}^{\prime}\right)} d C
$$


Distance $r(\boldsymbol{x}, \boldsymbol{\xi})$ between any point $\boldsymbol{x}$ in the domain and an arbitrary point $\boldsymbol{\xi}$ at the earthing electrode surface can be expressed as:

$$
r(\boldsymbol{x}, \boldsymbol{\xi})=\sqrt{|\boldsymbol{x}-\hat{\boldsymbol{\xi}}|^{2}+\frac{\phi^{2}(\hat{\boldsymbol{\xi}})}{4}-|\boldsymbol{x}-\hat{\boldsymbol{\xi}}| \phi(\hat{\boldsymbol{\xi}}) \sin \omega \cos \theta}
$$

where $\theta$ is the angular position in the perimeter of cross section of the cylindrical conductor, and

$$
\sin \omega=\frac{|(\hat{\boldsymbol{\xi}}-\boldsymbol{x}) \times \hat{\boldsymbol{s}}(\hat{\boldsymbol{\xi}})|}{|\hat{\boldsymbol{\xi}}-\boldsymbol{x}|}
$$

as shown in figure 1.

The elliptic integral obtained when $r(\boldsymbol{x}, \boldsymbol{\xi})$ in $(27)$ is substituted into (26) can be approximated by means of numerical integration up to an arbitrary level of accuracy. However, since we are interested in computing potential at points $\boldsymbol{x}$ on the earth surface, which distance to arbitrary points $\widehat{\boldsymbol{\xi}}$ on the axial lines is much larger that the diameter $\phi(\hat{\xi})$ of the earthing electrode [8], distance $r(\boldsymbol{x}, \boldsymbol{\xi})$ in $(27)$ can be approximated as

$$
r(\boldsymbol{x}, \boldsymbol{\xi}) \approx \hat{r}(\boldsymbol{x}, \hat{\boldsymbol{\xi}})=\sqrt{|\boldsymbol{x}-\hat{\boldsymbol{\xi}}|^{2}+\frac{\phi^{2}(\hat{\boldsymbol{\xi}})}{4}}
$$

Then, the inner integral of kernel $k(\boldsymbol{x}, \boldsymbol{\xi})$ in (26) can be approximated as:

$$
\int_{\boldsymbol{\xi} \in C(\widehat{\boldsymbol{\xi}})} k(\boldsymbol{x}, \boldsymbol{\xi}) d C \approx \pi \phi(\widehat{\boldsymbol{\xi}}) \hat{k}(\boldsymbol{x}, \widehat{\boldsymbol{\xi}})
$$

where

$$
\hat{k}(\boldsymbol{x}, \widehat{\boldsymbol{\xi}})=\left(\frac{1}{\widehat{r}(\boldsymbol{x}, \hat{\boldsymbol{\xi}})}+\frac{1}{\widehat{r}\left(\boldsymbol{x}, \hat{\boldsymbol{\xi}}^{\prime}\right)}\right)
$$

and $\hat{\boldsymbol{\xi}}^{\prime}$ is the symmetric of $\widehat{\boldsymbol{\xi}}$ with respect to the earth surface. Expression (30) can be interpreted as the result of applying a Newton-Cotes cuadrature with one single point to (26). This approximation will be quite accurate unless distance between points $\boldsymbol{x}$ and $\hat{\boldsymbol{\xi}}$ is of the same order of magnitude as diameter $\phi(\hat{\boldsymbol{\xi}})$, which will not occur if this approximation is used to compute potential values on the earth surface. 


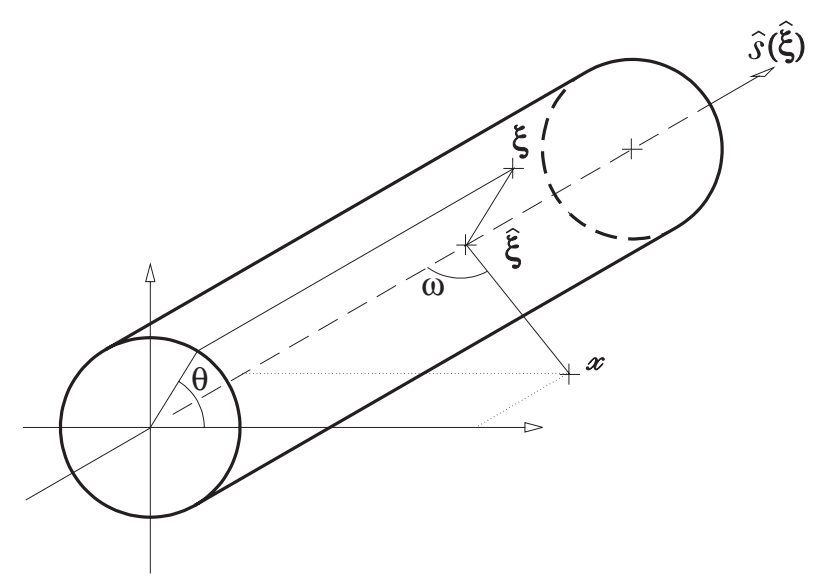

Fig. 1. Distance between a given point $\boldsymbol{x}$ and an arbitrary point $\boldsymbol{\xi}$ at the electrode surface.

Substituting (30) into (17), we can approximate:

$$
K(\hat{\chi}, \hat{\boldsymbol{\xi}}) \approx \int_{\boldsymbol{\chi} \in C(\hat{\boldsymbol{\chi}})} \pi \phi(\hat{\boldsymbol{\xi}}) \hat{k}(\chi, \hat{\boldsymbol{\xi}}) d C
$$

Next, bearing in mind once again the approximations used in (29), integral kernel (17) can now be simplified as:

$$
K(\hat{\chi}, \hat{\boldsymbol{\xi}}) \approx \pi \phi(\hat{\boldsymbol{\xi}}) \pi \phi(\hat{\boldsymbol{\chi}}) \hat{\hat{k}}(\hat{\boldsymbol{\chi}}, \hat{\boldsymbol{\xi}})
$$

being

$$
\hat{\hat{k}}(\hat{\boldsymbol{\chi}}, \hat{\boldsymbol{\xi}})=\left(\frac{1}{\hat{\hat{r}}(\hat{\boldsymbol{\chi}}, \hat{\boldsymbol{\xi}})}+\frac{1}{\hat{\hat{r}}\left(\hat{\boldsymbol{\chi}}, \hat{\boldsymbol{\xi}}^{\prime}\right)}\right),
$$

and

$$
\hat{\hat{r}}(\hat{\boldsymbol{\chi}}, \hat{\boldsymbol{\xi}})=\sqrt{|\hat{\boldsymbol{\chi}}-\hat{\boldsymbol{\xi}}|^{2}+\frac{\phi^{2}(\hat{\boldsymbol{\xi}})+\phi^{2}(\hat{\chi})}{4}}
$$

where inclusion of both diameters $\phi(\hat{\boldsymbol{\chi}})$ and $\phi(\hat{\boldsymbol{\xi}})$ authomatically preserves the symmetry in the system of equations (22) although the conductor cross sections were different at points $\hat{\boldsymbol{\chi}}$ and $\hat{\boldsymbol{\xi}}$.

Now, different selections of the sets of trial and test functions in (24) and (25) allow us to obtain specific formulations. Thus, for constant leakage current elements (one centered node per segment of conductor), Point Collocation (Dirac deltas as trial functions) leads to the very early intuitive methods, based 
on the idea that each segment of conductor is substituted for an "imaginary sphere". On the other hand, Galerkin type weighting (where test functions are identical to trial functions) leads to a kind of more recent methods (such as the "Average Potential Method, APM") [3], which were developed on the basic idea that each segment of conductor is substituted for a "line of point sources over the length of the conductor" [6] (constant leakage current elements). In these methods, coefficients (24) are usually referred as "mutual and self resistances" between "segments of conductor" [4].

The problems encountered with the application of these methods can now be explained from a mathematically rigorous point of view $[4,6]$. With the aim of studying the effect of simplifications (30) and (33), several numerical tests have been performed for a single bar in an infinite domain test problem [14]. This problem has been solved by means of 1) the simplified 1D boundary element formulation presented in this paper, 2) a 2D boundary element standard formulation for axisymmetrical potential problems (where no approximations are made in the integral equation kernel and circumferential integrals), and 3) a 2D Finite Element Method specific code for axisymmetrical potential problems.

The simplified 1D formulation results agree significantly with those obtained by the other two methods. However, as discretization level increases, oscillations around the real solution only occur in the $1 \mathrm{D}$ approach. Since the circumferential uniformity hypothesis is strictly satisfied in this case [14], and oscillations do not occur in the 2D boundary element standard formulation, the origin of these problems must be sought for in the simplifications introduced in the $1 \mathrm{D}$ approach.

The fact is that approximation (30) is not valid for short distances. Hence, when discretization is increased, and the conductor diameter becomes comparable to the size of the elements, approximation (33) introduces significant errors in the contributions (24) to the coefficients of the linear system (22) that correspond to adjacent nodes and specially in the diagonal terms.

From another point of view, since approximation error increases as discretization does, numerical results for dense discretizations do not trend to the solution of the integral equation (6) with kernel (5), but to the solution of a different ill-conditioned integral equation (18) with kernel $(33)[7,8]$.

This explains why unrealistic results are obtained when discretization increases [4], and convergence is precluded [7]. However, in the analysis of real grounding systems, results obtained for low and medium levels of discretization have been proved to be sufficiently accurate for practical purposes $[8,14]$.

Further discussion and examples are restricted to Galerkin type weighting, where the matrix of coefficients of linear system (22) is symmetric and positive 
definite [15]. Now, if diameter of conductors is assumed constant within each element, final expressions for computing potential coefficients (21) and linear system coefficients (24) can be written as

$$
\begin{aligned}
& \hat{V}_{i}^{\alpha}(\boldsymbol{x}) \approx \frac{\phi^{\alpha}}{4 \gamma} \int_{\hat{\boldsymbol{\xi}} \in L^{\alpha}} \hat{k}(\boldsymbol{x}, \hat{\boldsymbol{\xi}}) \widehat{N}_{i}(\hat{\boldsymbol{\xi}}) d L \\
& \hat{R}_{j i}^{\beta \alpha} \approx \frac{\pi \phi^{\beta} \phi^{\alpha}}{4 \gamma} \int_{\widehat{\boldsymbol{\chi}} \in L^{\beta}} \widehat{N}_{j}(\hat{\boldsymbol{\chi}})\left[\int_{\hat{\boldsymbol{\xi}} \in L^{\alpha}} \hat{\hat{k}}(\hat{\boldsymbol{\chi}}, \widehat{\boldsymbol{\xi}}) \widehat{N_{i}}(\widehat{\boldsymbol{\xi}}) d L\right] d L
\end{aligned}
$$

where $\phi^{\alpha}$ and $\phi^{\beta}$ represent the constant diameter within elements $L^{\alpha}$ and $L^{\beta}$. Obviously, contributions (37) lead to a symmetric matrix in (23).

Nevertheless, computation of the remaining integrals in (36) and (37) is not obvious. Gauss quadratures can not be used due to the undesirable behaviour of the integrands. Although very costly, a compound adaptative Simpson quadrature (with Richardson extrapolation error estimates) seems to be the best numerical choice [6]. Therefore, we turn our attention to analytical integration techniques.

Explicit formulae have been derived to compute (36) in the case of constant (1 functional node), linear (2 functional nodes) and parabolic (three functional nodes) leakage current elements [7]. Explicit expressions have also been recently derived for contributions (37). For the most simple cases these formulae reduce to those proposed in the literature (i.e. constant leakage current elements in APM [3]). Derivation of these formulae requires a large and not obvious, but systematic, analytical work, which is too cumbersome to be made completely explicit in this paper. In section $\mathbf{4}$, a summary of the whole development is presented.

\subsection{Overall Efficiency of the $1 D$ BEM Approach}

With regard to overall computational cost, for a given discretization ( $m$ elements of $p$ nodes each, and a total number of $n$ degrees of freedom) a linear system (22) of order $n$ must be generated and solved. Since the matrix is symmetric, but not sparse, resolution by means of a direct method requires $O\left(n^{3} / 3\right)$ operations. Matrix generation requires $O\left(m^{2} p^{2} / 2\right)$ operations (each one corresponding to a double integral), since $p^{2}$ contributions of type (37) have to be computed for every pair of elements, and approximately half of them are discarded because its symmetry.

Hence, most of computing efforts are devoted to matrix generation in sma$11 /$ medium problems, while linear system resolution prevails in medium/large 
ones. In these cases, the use of direct methods for the linear system resolution is out of range. Therefore iterative or semiiterative techniques will be preferable. The best results have been obtained by a diagonal preconditioned conjugate gradient algorithm with assembly of the global matrix [18], which has been implemented in a computer aided design system for grounding grids developed by the authors [19]. This technique has turned out to be extremely efficient for solving large scale problems, with a very low computational cost in comparison with the matrix generation effort.

At present, the size of the largest problem that can be solved with a conventional personal computer is limited by memory storage, required to record and handle the coefficients matrix. Thus, for a problem with 2000 degrees of freedom, at least $16 \mathrm{Mb}$ would be needed, while computing time for matrix generation and system resolution would be acceptable (but noticeable) and in the same order of magnitude (about half an hour on what is considered a medium performance Workstation or high performance PC in 1996).

On the other hand, once the leakage current has been obtained, the cost of computing the equivalent resistance (2) is negligible. The additional cost of computing potential at any given point (normally on the earth surface) by means of $(20)$ and (36) requires only $O(m p)$ operations. However, if it is necessary to compute potentials at a large number of points (i.e. to draw contours), computing time may also be important.

Selection of the type of leakage current density element is another important point in the resolution of a specific problem. Clearly, for a given discretization, constant density elements will provide less accurate results than linear or parabolic ones although with a low computational effort. Obviously, in comparison with the results obtained with a very crude grid of constant density elements, accuracy could be increased either rising the number of elements in the discretization, or using higher order elements (linear or parabolic) [14]. We must take into account that the obtention of asymptotical solutions by increasing the discretization level indefinitely is precluded, because approximations (30) and (33) are not valid if the size of elements becomes comparable to the electrode diameter as it was stated before. Thus, for a given problem it will be essential to consider the relative advantages and disadvantages of increasing the number of elements ( $h$ method, in the usual terminology of Finite Elements) or using higher order elements [8] ( $p$ method), in order to define an adecquate mesh. 


\section{Analytical Integration of Coefficients}

We present in this section the whole development of explicit formulae to compute analytically coefficients $\hat{V}_{i}^{\alpha}(\boldsymbol{x})$ in (36) and contributions $\hat{R}_{j i}^{\beta \alpha}$ in (37), which respectively correspond to the $i$-th trial function contribution to potential generated by element $\alpha$ at an arbitrary point $x$, and the $i$-th trial function contribution to potential generated by the element $\alpha$ over the surface of element $\beta$, weighted by the $j$-th test function.

In the further development, elements can be rectilinear segments (defined by their mid-point, length and axial unit vector), with an arbitrarily large number of functional nodes. However, in this paper we will only present the whole development for constant (1 functional node), linear (2 functional nodes) and parabolic (3 functional nodes) leakage current density elements.

\subsection{Computation of Potential Coefficients $\hat{V}_{i}^{\alpha}(\boldsymbol{x})$}

Any given point $\hat{\boldsymbol{\xi}} \in L^{\alpha}$ can be expressed in terms of the mid-point $\hat{\boldsymbol{\xi}}_{0}^{\alpha}$, the length $\mathcal{L}^{\alpha}$ and the axial unit vector $\hat{\boldsymbol{s}}^{\alpha}$, for a value of the scalar parameter $\xi$ varying within the range -1 to 1 (domain of isoparametric $1 D$ shape functions) [16], in the standard form

$$
\widehat{\boldsymbol{\xi}}(\xi)=\hat{\boldsymbol{\xi}}_{0}^{\alpha}+\xi \frac{\mathcal{L}^{\alpha}}{2} \widehat{\boldsymbol{s}}^{\alpha}
$$

Thus, (36) can be rewritten as the line integral

$$
\hat{V}_{i}^{\alpha}(\boldsymbol{x})=\frac{\phi^{\alpha} \mathcal{L}^{\alpha}}{8 \gamma} \int_{\xi=-1}^{\xi=1} \hat{k}(\boldsymbol{x}, \widehat{\xi}(\xi)) \widehat{N}_{i}(\widehat{\xi}(\xi)) d \xi
$$

Then, it is possible to express the integral kernel $\hat{k}(\boldsymbol{x}, \widehat{\boldsymbol{\xi}}(\xi))$ as a direct function of $\xi$, since distance $\hat{r}(\boldsymbol{x}, \hat{\boldsymbol{\xi}}(\xi))$ results in

$$
\widehat{r}(\boldsymbol{x}, \widehat{\boldsymbol{\xi}}(\xi))=\frac{\mathcal{L}^{\alpha}}{2} \sqrt{\left(\hat{p}^{\alpha}(\boldsymbol{x})\right)^{2}+\left(\hat{q}^{\alpha}(\boldsymbol{x})-\xi\right)^{2}},
$$

where

$$
\left(\hat{p}^{\alpha}(\boldsymbol{x})\right)^{2}=\left(\frac{p^{\alpha}(\boldsymbol{x})}{\mathcal{L}^{\alpha} / 2}\right)^{2}+\left(\frac{\phi^{\alpha}}{\mathcal{L}^{\alpha}}\right)^{2}, \quad \hat{q}^{\alpha}(\boldsymbol{x})=\frac{q^{\alpha}(\boldsymbol{x})}{\mathcal{L}^{\alpha} / 2},
$$


and being $p^{\alpha}(\boldsymbol{x})$ the distance between the point $\boldsymbol{x}$ and its orthogonal projection over the axial line of the electrode, and $q^{\alpha}(\boldsymbol{x})$ the relative position between the previously mentioned orthogonal projection and the mid-point $\hat{\boldsymbol{\xi}}_{0}^{\alpha}$,

$$
p^{\alpha}(\boldsymbol{x})=\left|\boldsymbol{x}-\hat{\boldsymbol{\xi}}_{0}^{\alpha}-q^{\alpha}(\boldsymbol{x}) \hat{\boldsymbol{s}}^{\alpha}\right|, \quad q^{\alpha}(\boldsymbol{x})=\left(\boldsymbol{x}-\hat{\boldsymbol{\xi}}_{0}^{\alpha}\right) \cdot \hat{\boldsymbol{s}}^{\alpha}
$$

Analogous expressions in terms of the corresponding geometrical parameters $\hat{p}^{\alpha \prime}(\boldsymbol{x})$ and $\hat{q}^{\alpha \prime}(\boldsymbol{x})$ can be easily obtained for the image distance $\widehat{r}\left(\boldsymbol{x}, \hat{\boldsymbol{\xi}}^{\prime}(\xi)\right)$ in $(31)$,

$$
\left(\hat{p}^{\alpha \prime}(\boldsymbol{x})\right)^{2}=\left(\frac{p^{\alpha \prime}(\boldsymbol{x})}{\mathcal{L}^{\alpha} / 2}\right)^{2}+\left(\frac{\phi^{\alpha}}{\mathcal{L}^{\alpha}}\right)^{2}, \quad \hat{q}^{\alpha \prime}(\boldsymbol{x})=\frac{q^{\alpha \prime}(\boldsymbol{x})}{\mathcal{L}^{\alpha} / 2},
$$

where

$$
p^{\alpha \prime}(\boldsymbol{x})=\left|\boldsymbol{x}-\widehat{\boldsymbol{\xi}}_{0}^{\alpha \prime}-q^{\alpha^{\prime}}(\boldsymbol{x}) \hat{\boldsymbol{s}}^{\alpha \prime}\right|, \quad q^{\alpha \prime}(\boldsymbol{x})=\left(\boldsymbol{x}-\hat{\boldsymbol{\xi}}_{0}^{\alpha \prime}\right) \cdot \hat{\boldsymbol{s}}^{\alpha \prime},
$$

being $\hat{\boldsymbol{\xi}}_{0}^{\alpha \prime}$ the mid-point and $\hat{\boldsymbol{s}}^{\alpha \prime}$ the axial unit vector of the image of $L^{\alpha}$.

On the other hand, any constant, linear or parabolic shape function $\widehat{N}_{i}(\hat{\boldsymbol{\xi}}(\xi))$ in (39) can be approximated - by means of their Taylor series expansion up to the second order term - as a parabolic function in the variable $\xi$

$$
\widetilde{N}_{i}(\widehat{\boldsymbol{\xi}}(\xi))=\widehat{n}_{0 i}+\widehat{n}_{1 i} \xi+\widehat{n}_{2 i} \xi^{2}
$$

which coefficients $\left(\widehat{n}_{0 i}, \hat{n}_{1 i}, \hat{n}_{2 i}\right)$ depend on the nodal positions.

Finally, if we substitute (40) in (31) and (45) in (39) it is possible to integrate explicitly the potential coefficient $\hat{V}_{i}^{\alpha}(\boldsymbol{x})$. Thus, after a relatively large analytical development, (39) can be expressed as

$$
\hat{V}_{i}^{\alpha}(\boldsymbol{x})=\frac{\phi^{\alpha}}{4 \gamma}\left[\Phi\left(\hat{p}^{\alpha}(\boldsymbol{x}), \hat{q}^{\alpha}(\boldsymbol{x})\right)+\Phi\left(\hat{p}^{\alpha \prime}(\boldsymbol{x}), \hat{q}^{\alpha \prime}(\boldsymbol{x})\right)\right]
$$

where function $\Phi(\cdot, \cdot)$ depends only on geometrical parameters $\hat{p}^{\alpha}(\boldsymbol{x}), \hat{q}^{\alpha}(\boldsymbol{x})$ and known coefficients of the shape functions [8]. Explicit expressions for $\Phi\left(\hat{p}^{\alpha}(\boldsymbol{x}), \hat{q}^{\alpha}(\boldsymbol{x})\right)$ are given in appendix 1 .

\subsection{Computation of System Coefficients $\hat{R}_{j i}^{\beta \alpha}$}

Any given point $\hat{\chi} \in L^{\beta}$ can be expressed in terms of the mid-point $\hat{\chi}_{0}^{\beta}$, the length $\mathcal{L}^{\beta}$ and the axial unit vector $\hat{\boldsymbol{s}}^{\beta}$, for a value of scalar parameter $\chi$ varying 
within the range -1 to 1 (domain of isoparametric $1 \mathrm{D}$ shape functions) [16], in the form

$$
\hat{\chi}(\chi)=\hat{\boldsymbol{\chi}}_{0}^{\beta}+\chi \frac{\mathcal{L}^{\beta}}{2} \hat{\boldsymbol{s}}^{\beta}
$$

Thus, taking into account the development achieved in (39), expression (37) can be rewritten in terms of two line integrals,

$$
\widehat{R}_{j i}^{\beta \alpha}=\frac{\pi \phi^{\alpha} \phi^{\beta} \mathcal{L}^{\alpha} \mathcal{L}^{\beta}}{16 \gamma}\left\{\int_{\chi=-1}^{\chi=1} \widehat{N}_{j}(\hat{\boldsymbol{\chi}}(\chi))\left[\int_{\xi=-1}^{\xi=1} \widehat{\hat{k}}(\hat{\boldsymbol{\chi}}(\chi), \widehat{\boldsymbol{\xi}}(\xi)) \widehat{N}_{i}(\widehat{\boldsymbol{\xi}}(\xi)) d \xi\right] d \chi\right\}
$$

It may be seen that the line integral on $\xi$ in (48) is similar to the line integral in (39), although in (48) the integral kernel is given by (34). Then, (48) can be expressed, by means of (46), as

$$
\begin{aligned}
& \hat{R}_{j i}^{\beta \alpha}=\frac{\pi \phi^{\alpha} \phi^{\beta} \mathcal{L}^{\beta}}{8 \gamma}\left\{\quad \widehat{\mathcal{R}}_{j i}^{\beta \alpha}\left(\widehat{\boldsymbol{\xi}}_{0}^{\alpha}, \hat{\boldsymbol{s}}^{\alpha}, \mathcal{L}^{\alpha}, \hat{\boldsymbol{\chi}}_{0}^{\beta}, \hat{\boldsymbol{s}}^{\beta}, \mathcal{L}^{\beta}\right)\right. \\
& \left.+\widehat{\mathcal{R}}_{j i}^{\beta \alpha}\left(\widehat{\boldsymbol{\xi}}_{0}^{\alpha \prime}, \widehat{\boldsymbol{s}}^{\alpha \prime}, \mathcal{L}^{\alpha}, \hat{\boldsymbol{\chi}}_{0}^{\beta}, \hat{\boldsymbol{s}}^{\beta}, \mathcal{L}^{\beta}\right)\right\},
\end{aligned}
$$

being

$$
\widehat{\mathcal{R}}_{j i}^{\beta \alpha}\left(\hat{\boldsymbol{\xi}}_{0}^{\alpha}, \hat{\boldsymbol{s}}^{\alpha}, \mathcal{L}^{\alpha}, \hat{\boldsymbol{\chi}}_{0}^{\beta}, \hat{\boldsymbol{s}}^{\beta}, \mathcal{L}^{\beta}\right)=\int_{\chi=-1}^{\chi=1} \widehat{N}_{j}(\widehat{\boldsymbol{\chi}}(\chi))\left[\Phi\left(\hat{r}^{\alpha}(\widehat{\chi}(\chi)), \widehat{q}^{\alpha}(\widehat{\boldsymbol{\chi}}(\chi))\right)\right] d \chi
$$

and

$$
\begin{array}{r}
\left(\hat{r}^{\alpha}(\hat{\boldsymbol{\chi}}(\chi))\right)^{2}=\left(\frac{p^{\alpha}(\hat{\boldsymbol{\chi}}(\chi))}{\mathcal{L}^{\alpha} / 2}\right)^{2}+\left(\frac{\phi^{\alpha}}{\mathcal{L}^{\alpha}}\right)^{2}+\left(\frac{\phi^{\beta}}{\mathcal{L}^{\alpha}}\right)^{2} \\
\hat{q}^{\alpha}(\hat{\boldsymbol{\chi}}(\chi))=\frac{q^{\alpha}(\hat{\boldsymbol{\chi}}(\chi))}{\mathcal{L}^{\alpha} / 2}
\end{array}
$$

where functions $p^{\alpha}(\cdot)$ and $q^{\alpha}(\cdot)$ are given by (42). Analogous expressions for the image term in (49) 


$$
\widehat{\mathcal{R}}_{j i}^{\beta \alpha}\left(\hat{\boldsymbol{\xi}}_{0}^{\alpha \prime}, \hat{\boldsymbol{s}}^{\alpha \prime}, \mathcal{L}^{\alpha}, \hat{\boldsymbol{\chi}}_{0}^{\beta}, \hat{\boldsymbol{s}}^{\beta}, \mathcal{L}^{\beta}\right) \int_{\chi=-1}^{\chi=1} \widehat{N}_{j}(\hat{\chi}(\chi))\left[\Phi\left(\hat{r}^{\alpha \prime}(\widehat{\chi}(\chi)), \hat{q}^{\alpha \prime}(\widehat{\boldsymbol{\chi}}(\chi))\right)\right] d \chi,
$$

can be easily obtained in terms of the corresponding geometrical parameters $\hat{r}^{\alpha \prime}(\hat{\chi}(\chi))$ and $\hat{q}^{\alpha \prime}(\hat{\chi}(\chi))$,

$$
\begin{array}{r}
\left(\hat{r}^{\alpha \prime}(\hat{\boldsymbol{\chi}}(\chi))\right)^{2}=\left(\frac{p^{\alpha \prime}(\hat{\boldsymbol{\chi}}(\chi))}{\mathcal{L}^{\alpha} / 2}\right)^{2}+\left(\frac{\phi^{\alpha}}{\mathcal{L}^{\alpha}}\right)^{2}+\left(\frac{\phi^{\beta}}{\mathcal{L}^{\alpha}}\right)^{2} \\
\hat{q}^{\alpha \prime}(\hat{\boldsymbol{\chi}}(\chi))=\frac{q^{\alpha^{\prime}}(\hat{\boldsymbol{\chi}}(\chi))}{\mathcal{L}^{\alpha} / 2}
\end{array}
$$

where fucntions $p^{\alpha^{\prime}}(\cdot)$ and $q^{\alpha^{\prime}}(\cdot)$ are given by (44).

On the other hand, any constant, linear or parabolic shape function $\widehat{N}_{j}(\widehat{\chi}(\chi))$ in (50) and (52) can be approximated - by means of their Taylor series expansion up to the second order term - as a parabolic function in the variable $\chi$

$$
\widetilde{N}_{j}(\hat{\chi}(\chi))=\widehat{n}_{0 j}+\widehat{n}_{1 j} \chi+\widehat{n}_{2 j} \chi^{2}
$$

which coefficients $\left(\widehat{n}_{0 j}, \widehat{n}_{1 j}, \hat{n}_{2 j}\right)$ depend on the nodal positions.

Now, if we substitute (51) and (54) in (50), we obtain a line integral in the variable $\chi$ for the term $\widehat{\mathcal{R}}_{j i}^{\beta \alpha}\left(\hat{\boldsymbol{\xi}}_{0}^{\alpha}, \widehat{\boldsymbol{s}}^{\alpha}, \mathcal{L}^{\alpha}, \hat{\boldsymbol{\chi}}_{0}^{\beta}, \hat{\boldsymbol{s}}^{\beta}, \mathcal{L}^{\beta}\right)$, which is possible to integrate explicitly.

This explicit integration requires previously a geometrical analysis of the two rectilinear segments $-\alpha$ and $\beta$ - in the space. This study allows to express terms $\hat{r}^{\alpha}(\hat{\chi}(\chi))$ and $\hat{q}^{\alpha}(\hat{\chi}(\chi))$ in $(50)$ as functions of the variable $\chi$ and a set of geometrical parameters depending on the relative position between segments $[8]$.

If we finally substitute expressions obtained for $\hat{r}^{\alpha}(\hat{\chi}(\chi))$ and $\hat{q}^{\alpha}(\hat{\chi}(\chi))$ and the shape function $\widehat{N}_{j}(\hat{\chi}(\chi)),(50)$ can be rewritten - after suitable arrangements-as:

$$
\widehat{\mathcal{R}}_{j i}^{\beta \alpha}\left(\hat{\boldsymbol{\xi}}_{0}^{\alpha}, \hat{\boldsymbol{s}}^{\alpha}, \mathcal{L}^{\alpha}, \hat{\boldsymbol{\chi}}_{0}^{\beta}, \hat{\boldsymbol{s}}^{\beta}, \mathcal{L}^{\beta}\right)=\sum_{u=0}^{u=2} \sum_{w=0}^{w=4} \mathcal{K}_{w}^{(u)} \varphi_{w}^{(u)}
$$

where coefficients $\mathcal{K}_{w}^{(u)}$ can be directly computed from the $j$-th shape function, the geometrical parameters of electrodes and the $i$-th shape function [8]. On the other hand, all line integrals in the variable $\chi$ can be incorporated in 
coefficients $\varphi_{w}^{(u)}$ in (55). Specific expressions for these terms can be found in appendix 2 .

Obviously, analogous coefficients for the image term (52) can be easily obtained from the analysis of parameters $\hat{r}^{\alpha \prime}(\hat{\chi}(\chi))$ and $\hat{q}^{\alpha \prime}(\hat{\chi}(\chi))$.

The obtention of explicit formulae to evaluate expressions $\varphi_{w}^{(u)}$ is not obvious, and requires quite a lot of analytical work. Since these coefficients also depend on the geometrical parameters of electrodes, we must analyze an important number of different type of integrals due to the extense casuistry [8].

At the beginning of this research [17], analytical expressions were derived only for the simplest spatial arrangements of electrodes (perpendicular and parallel bars). At present the whole development has been completed, and analytical expressions have been obtained to compute all coefficients $\varphi_{w}^{(u)}$.

We remark that these analytical formulae have been obtained taking into account their latter implementation in a computer code. Special attention has been devoted to obtain recurrent forms, using the minimum number of operations involving trascendental functions. Anyway, the final implementation in a computer code must be done with care, in order to avoid numerical illconditioning due to round-off errors.

A summary of these expressions is given in appendix 2. The complete derivation (which is too cumbersome to be made explicit in this paper) can be found in previous literature [8].

\section{Application to Real Cases}

The first example that we present is the E. R. Barberá substation grounding operated by the power company Fecsa, close to the city of Barcelona in Spain. The earthing system of this substation is a grid of 408 cylindrical conductors with constant diameter $(12.85 \mathrm{~mm})$ buried to a depth of $80 \mathrm{~cm}$, being the total surface protected up to $6500 \mathrm{~m}^{2}$. The total area studied is a rectangle of $135 \mathrm{~m}$ by $210 \mathrm{~m}$, which implies a surface up to $28000 \mathrm{~m}^{2}$. The Ground Potential Rise considered in this study is $10 \mathrm{kV}$ (due to the linear relation between the Ground Potential Rise $V_{\Gamma}$ and the Total Surge Current $I_{\Gamma}$, we can consider one as given and the other as unknown or viceversa). The plan of the grounding grid and its characteristics are presented in figure 2 and table 1 .

The numerical model used in the resolution of this problem is based on a Galerkin type weighting. Each bar is discretized in one single linear leakage 
Table 1

E. R. Barberá Substation: Characteristics.

\begin{tabular}{|c|c|}
\hline \multicolumn{2}{|c|}{ E. R. BARBERÁ GROUNDING SYSTEM } \\
\hline Max. Grid Dimensions: & $145 \mathrm{~m} \times 90 \mathrm{~m}$ \\
Grid Depth: & $0.80 \mathrm{~m}$ \\
Number of Grid Electrodes: & 408 \\
Electrode Diameter: & $12.85 \mathrm{~mm}$ \\
Ground Potential Rise: & $10 \mathrm{kV}$ \\
Earth Resistivity: & $60 \Omega \mathrm{m}$ \\
\hline
\end{tabular}

Table 2

E.R. Barberá Substation: Numerical Model and BEM Results.

\section{E. R. BARBERÁ GROUNDING SYSTEM: 1D BEM MODEL \& RESULTS}

\begin{tabular}{|c|c|}
\hline Type of Element: & Linear \\
Number of Elements: & 408 \\
Degrees of Freedom: & 238 \\
Fault Current: & $31.8 \mathrm{kA}$ \\
Equivalent Resistance: & $0.315 \Omega$ \\
CPU Time: & $450 \mathrm{~s}$ \\
Computer: & $\mathrm{PC} 486 / 16 \mathrm{Mb} / 66 \mathrm{MHz}$ \\
\hline
\end{tabular}

current density element, which implies a total of 238 degrees of freedom ${ }^{1}$.

In this example, it can be shown that using linear elements reduces significantly the total number of degrees of freedom. Then, for a higher computational cost in matrix generation, and a lower computational cost in linear solving, higher precision results are obtained for a similar overall computing effort.

Results are given in table 2. Figure 2 shows the potential distribution on ground surface when a fault condition occurs, and potential profiles along different lines are represented in figure 3.

Numerical resolution of this model of the grounding grid has only required seven and a half minutes of CPU time in a conventional personal computer (i.e. $\mathrm{PC} 486 / 16 \mathrm{Mb}$ to $66 \mathrm{MHz}$ ).

\footnotetext{
${ }^{1}$ It is interesting to notice that using one single constant leakage current density element per electrode, the number of degrees of freedom in this example would be 408 .
} 

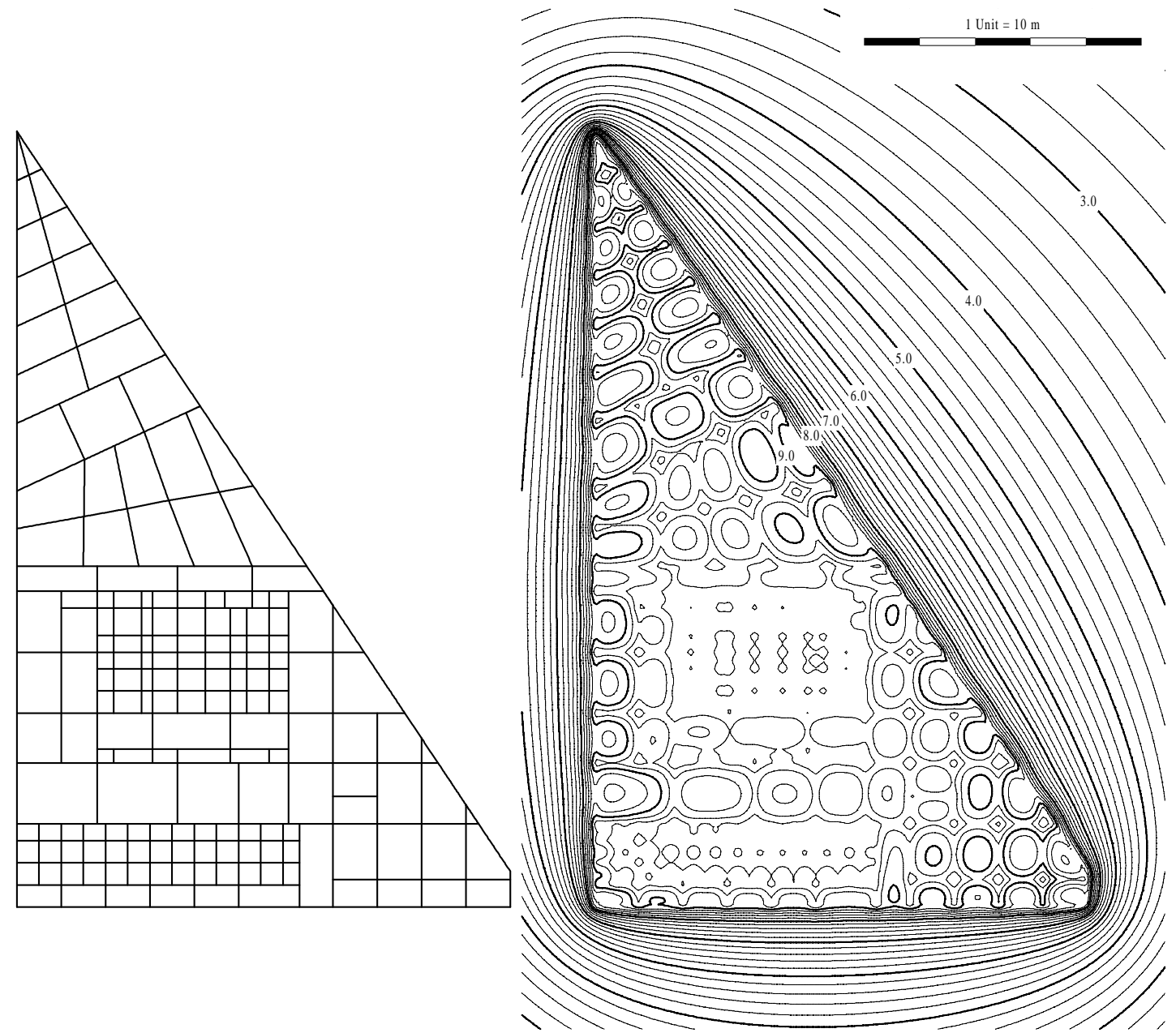

Fig. 2. E. R. Barberá Grounding Grid: Plan and Potential Distribution on Ground Surface (potential contours plotted every $0.2 \mathrm{kV}$, and thick contours every $1 \mathrm{kV}$ ).

The second example that we present is the Balaidos II substation grounding operated by the power company Unión Fenosa, close to the city of Vigo in Spain. The earthing system of this substation is a grid of 107 cylindrical conductors (diameter: $11.28 \mathrm{~mm}$ ) buried to a depth of $80 \mathrm{~cm}$, supplemented with 67 vertical rods (each one has a length of $2.5 \mathrm{~m}$ and a diameter of $14.0 \mathrm{~mm}$ ). The total surface protected up to $4800 \mathrm{~m}^{2}$. The total area studied is a rectangle of $121 \mathrm{~m}$ by $108 \mathrm{~m}$, which implies a surface up to $13000 \mathrm{~m}^{2}$. As in the previous case, the Ground Potential Rise considered in this study has been $10 \mathrm{kV}$. The plan of the grounding grid and its characteristics are presented in figure 4 and table 3 .

The numerical model used in the resolution of this problem is also based on a Galerkin type weighting. Each bar is now discretized in one single parabolic leakage current density element, which implies a total of 315 degrees of free- 

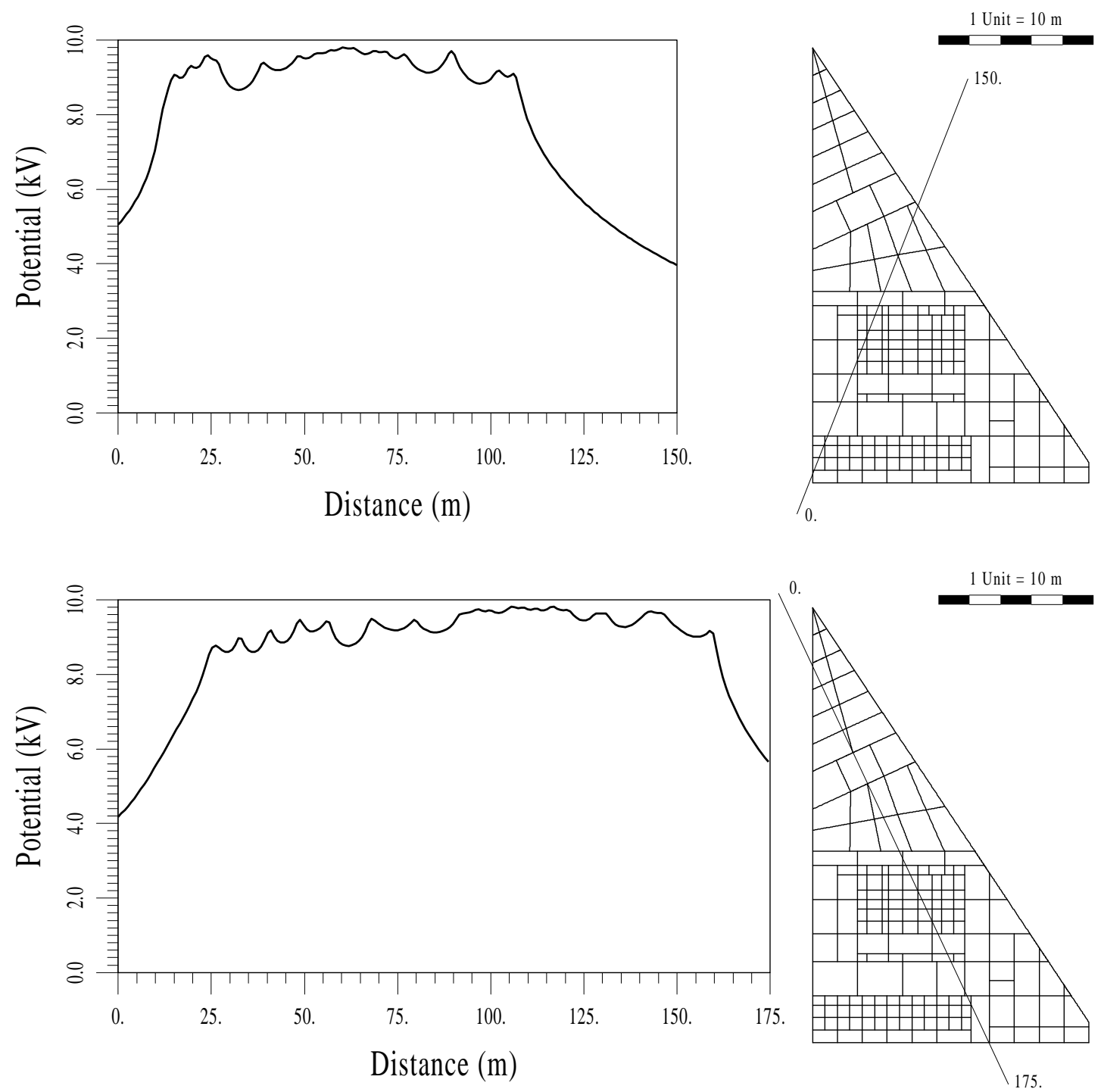

Fig. 3. E.R. Barbera Grounding Grid: Potential profiles along different lines. dom ${ }^{2}$.

Results are given in table 4 . Figure 4 shows the potential distribution on ground surface when a fault condition occurs.

Numerical resolution of this model has only required ten minutes of CPU time in a conventional personal computer (i.e. $\mathrm{PC} 486 / 16 \mathrm{Mb}$ to $66 \mathrm{MHz}$ ).

It can be shown in this example that using parabolic elements increases the total number of degrees of freedom. Obviously, computational cost devoted to matrix generation and linear solving is also increased, but the overall com-

\footnotetext{
${ }^{2}$ In this case, the use of one single constant density element per electrode would imply a total of 174 degrees of freedom, while the use of one single linear element would imply 141 degrees of freedom.
} 
Table 3

Balaidos II Substation: Characteristics.

\begin{tabular}{|c|c|}
\hline \multicolumn{2}{|c|}{ BALAIDOS II GROUNDING SYSTEM } \\
\hline Max. Grid Dimensions: & $60 \mathrm{~m} \times 80 \mathrm{~m}$ \\
Grid Depth: & $0.80 \mathrm{~m}$ \\
Number of Grid Electrodes: & 107 \\
Number of Vertical Rods: & 67 \\
Electrode Diameter: & $11.28 \mathrm{~mm}$ \\
Vertical Rod Diameter: & $14.00 \mathrm{~mm}$ \\
Ground Potential Rise: & $10 \mathrm{kV}$ \\
Earth Resistivity: & $60 \Omega \mathrm{m}$ \\
\hline
\end{tabular}
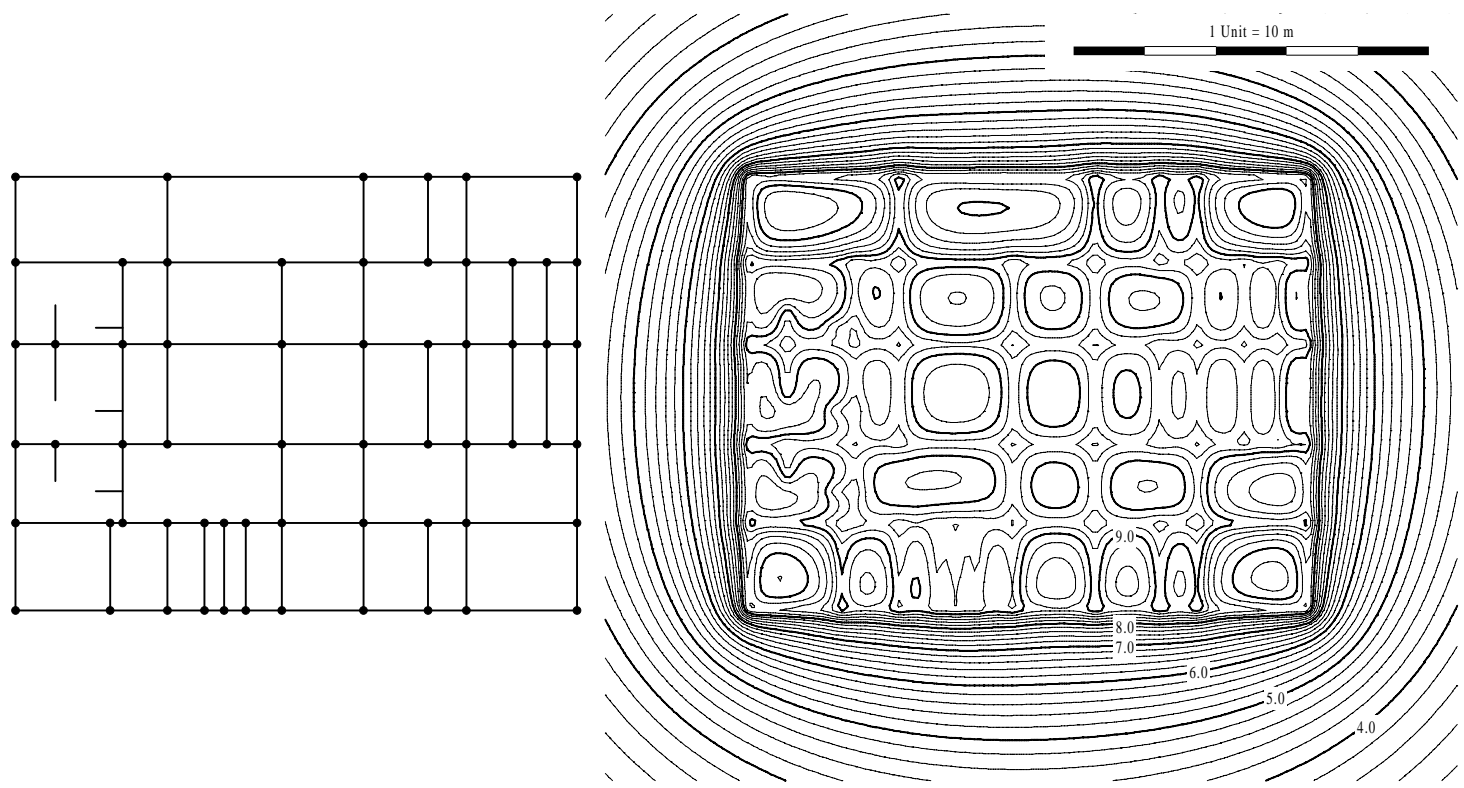

Fig. 4. Balaidos II Grounding Grid: Plan (vertical rods marked with black points) and Potential Distribution on Ground Surface (potential contours plotted every $0.2 \mathrm{kV}$, and thick contours every $1 \mathrm{kV}$ ).

puting effort is acceptable (of the same order of magnitude) while precision is much higher.

Both examples have been repeatedly solved increasing the segmentation of the electrodes. At the scale of the whole grid, results and potential distributions on the earth surface were not noticeably improved by increasing segmentation. We conclude, as a general rule, that a reasonable (moderate) level of segmentation is sufficient for practical purposes. Increasing the number of elements beyond this point will not be necessary unless high accuracy local results must be obtained for a limited part of the whole earthing system. And 
Table 4

Balaidos II Substation: Numerical Model and BEM Results.

\begin{tabular}{|c|c|}
\hline \multicolumn{2}{|c|}{ BALAIDOS II GROUNDING SYSTEM: } \\
1D BEM MODEL \& RESULTS \\
\hline Type of Element: & Parabolic \\
Number of Elements: & 174 \\
Degrees of Freedom: & 315 \\
Fault Current: & $25 \mathrm{kA}$ \\
Equivalent Resistance: & $0.4 \Omega$ \\
CPU Time: & $600 \mathrm{~s}$ \\
Computer: & PC $486 / 16 \mathrm{Mb} / 66 \mathrm{MHz}$ \\
\hline
\end{tabular}

finally, the use of higher order elements will be more advantageous (in general) than increasing segmentation intensively, since accuracy will be higher for a remarkably smaller total number of degrees of freedom [8].

\section{Conclusions}

A Boundary Element approach for the analysis of substation earthing systems has been presented. For 3D problems, some reasonable assumptions allow us to reduce a general 2D BEM formulation to an approximated less expensive $1 \mathrm{D}$ version. By means of new advanced integration techniques, it is possible to compute analytically all the coefficients of the numerical model, and reduce computing requirements in memory storage and CPU time under acceptable levels.

Several widespread intuitive methods can be identified as the result of specific choices for the test and trial functions and suitable assumptions introduced in the BEM formulation to reduce computational cost. Problems encountered with the application of these methods can now be finally explained from a mathematically rigorous point of view, while more efficient and accurate formulations can be derived.

Nowadays, all these techniques derived by the authors have allowed to develop a Computer Aided Design system (TOTBEM) [19] for earthing grids of electrical substations. With this system, it is possible to analyze accurately grounding grids of medium/big sizes, nearly in real time and using a low cost and widely available conventional computer. Obviously, the study of a larger installation should require a higher computing effort, and probably a more 
powerful computer, although the rise of the computational cost should be acceptable.

\section{Acknowledgements}

This work has been partially supported by power company "Unión Fenosa", by research fellowships of the "Universidad de La Coruña" and the regional government "Xunta de Galicia", and by a research project of power company "Fecsa".

Appendix 1. The analytical expression for function $\Phi(a, b)$ in $(46)$ can be expressed as [8]

$$
\Phi(a, b)=\mathcal{V}^{(0)} \varphi^{(0)}+\mathcal{V}^{(1)} \varphi^{(1)}+\mathcal{V}^{(2)} \varphi^{(2)},
$$

where coefficients $\left(\mathcal{V}^{(0)}, \mathcal{V}^{(1)}, \mathcal{V}^{(2)}\right)$ depend on geometrical parameters of the electrode and known values of the shape function:

$$
\mathcal{V}^{(0)}=\hat{n}_{0 i}+\hat{n}_{1 i} b+\hat{n}_{2 i}\left(b^{2}-a^{2} / 2\right), \quad \mathcal{V}^{(1)}=\hat{n}_{1 i}+2 b \hat{n}_{2 i}, \quad \mathcal{V}^{(2)}=\hat{n}_{2 i} / 2,
$$

and functions $\varphi^{(0)}, \varphi^{(1)}, \varphi^{(2)}$ are

$$
\begin{aligned}
& \varphi^{(0)}=\operatorname{ArgSh}\left(\frac{1-b}{a}\right)+\operatorname{ArgSh}\left(\frac{1+b}{a}\right) \\
& \varphi^{(1)}=\sqrt{(1-b)^{2}+a^{2}}-\sqrt{(1+b)^{2}+a^{2}} \\
& \varphi^{(2)}=(1-b) \sqrt{(1-b)^{2}+a^{2}}+(1+b) \sqrt{(1+b)^{2}+a^{2}}
\end{aligned}
$$

Appendix 2. Derivation of analytical expressions for computing coefficients in (55) requires a previous geometrical analysis of the relative position of two arbitrary elements $\alpha$ and $\beta$ in the 3D space, so that all the variables involved in $\widehat{\mathcal{R}}_{j i}^{\beta \alpha}\left(\hat{\boldsymbol{\xi}}_{0}^{\alpha}, \hat{\boldsymbol{s}}^{\alpha}, \mathcal{L}^{\alpha}, \hat{\boldsymbol{\chi}}_{0}^{\beta}, \hat{\boldsymbol{s}}^{\beta}, \mathcal{L}^{\beta}\right)$ can be expressed in terms of parameters that depend on the relative position between them. Thus, since we are considering elements formed by rectilinear segments, we can take a coordinates system which origin is the mid-point of $\alpha$ and the $y$-axis is placed on this segment. In this coordinates system, element $\beta$ can now be defined by a new mid-point $\tilde{\chi}_{0}^{\beta} \equiv\left(\tilde{\chi}_{0 x}^{\beta}, \tilde{\chi}_{0 y}^{\beta}, \tilde{\chi}_{0 z}^{\beta}\right)$ and a new axial unit vector $\tilde{\boldsymbol{s}}^{\beta} \equiv\left(\tilde{s}_{x}^{\beta}, \tilde{s}_{y}^{\beta}, \tilde{s}_{z}^{\beta}\right)$. 
In the further development, we introduce a set of parameters which contain the geometrical information between the two segments in terms of $\tilde{\boldsymbol{\chi}}_{0}^{\beta}, \tilde{\boldsymbol{s}}^{\beta}$ and lengths $\mathcal{L}^{\alpha}$ and $\mathcal{L}^{\beta}$. These quantities are

$$
\begin{aligned}
& \lambda=\frac{\mathcal{L}^{\beta}}{\mathcal{L}^{\alpha}}, \quad \mathcal{A}=\left(\frac{\tilde{\chi}_{0 y}}{\mathcal{L}^{\beta} / 2}\right), \quad \mathcal{B}=\tilde{s}_{y}^{\beta} \\
& \mathcal{C}^{2}=\left(\frac{\tilde{\chi}_{0 x}}{\mathcal{L}^{\beta} / 2}\right)^{2}+\left(\frac{\tilde{\chi}_{0 z}}{\mathcal{L}^{\beta} / 2}\right)^{2}+\left(\frac{\sqrt{\left(\phi^{\alpha} / 2\right)^{2}+\left(\phi^{\beta} / 2\right)^{2}}}{\mathcal{L}^{\beta} / 2}\right)^{2} \\
& \mathcal{D}=\left(\frac{\tilde{\chi}_{0 x}}{\mathcal{L}^{\beta} / 2}\right) \tilde{s}_{x}^{\beta}+\left(\frac{\tilde{\chi}_{0 z}}{\mathcal{L}^{\beta} / 2}\right) \tilde{s}_{z}^{\beta}, \quad \mathcal{E}^{2}=\left(\tilde{s}_{x}^{\beta}\right)^{2}+\left(\tilde{s}_{z}^{\beta}\right)^{2}
\end{aligned}
$$

Now, coefficients $\mathcal{K}_{w}^{(u)}$ in $(55)$ can be computed, in terms of $\lambda, \mathcal{A}, \mathcal{B}, \mathcal{C}, \mathcal{D}, \mathcal{E}$ and known values of shape functions, as

$$
\left(\begin{array}{l}
\mathcal{K}_{0}^{(0)} \\
\mathcal{K}_{1}^{(0)} \\
\mathcal{K}_{2}^{(0)} \\
\mathcal{K}_{3}^{(0)} \\
\mathcal{K}_{4}^{(0)}
\end{array}\right)=\left(\begin{array}{ccc}
\hat{n}_{0 j} & 0 & 0 \\
\hat{n}_{1 j} & \hat{n}_{0 j} & 0 \\
\hat{n}_{2 j} & \hat{n}_{1 j} & \hat{n}_{0 j} \\
0 & \hat{n}_{2 j} & \hat{n}_{1 j} \\
0 & 0 & \hat{n}_{2 j}
\end{array}\right)\left(\begin{array}{ccc}
1 & \lambda \mathcal{A} & \lambda^{2}\left(\mathcal{A}^{2}-\mathcal{C}^{2} / 2\right) \\
0 & \lambda \mathcal{B} & \lambda^{2}(2 \mathcal{A B}-\mathcal{D}) \\
0 & 0 & \lambda^{2}\left(\mathcal{B}^{2}-\mathcal{E}^{2} / 2\right)
\end{array}\right)\left(\begin{array}{c}
\hat{n}_{0 i} \\
\hat{n}_{1 i} \\
\hat{n}_{2 i}
\end{array}\right)
$$

$$
\left(\begin{array}{l}
\mathcal{K}_{0}^{(1)} \\
\mathcal{K}_{1}^{(1)} \\
\mathcal{K}_{2}^{(1)} \\
\mathcal{K}_{3}^{(1)} \\
\mathcal{K}_{4}^{(1)}
\end{array}\right)=\left(\begin{array}{cc}
\widehat{n}_{0 j} & 0 \\
\widehat{n}_{1 j} & \widehat{n}_{0 j} \\
\widehat{n}_{2 j} & \widehat{n}_{1 j} \\
0 & \widehat{n}_{2 j} \\
0 & 0
\end{array}\right)\left(\begin{array}{cc}
1 & 2 \lambda \mathcal{A} \\
0 & 2 \lambda \mathcal{B}
\end{array}\right)\left(\begin{array}{c} 
\\
\hat{n}_{1 i} \\
\hat{n}_{2 i}
\end{array}\right) ; \quad\left(\begin{array}{c}
\mathcal{K}_{0}^{(2)} \\
\mathcal{K}_{1}^{(2)} \\
\mathcal{K}_{2}^{(2)} \\
\mathcal{K}_{3}^{(2)} \\
\mathcal{K}_{4}^{(2)}
\end{array}\right)=\left(\begin{array}{c}
\hat{n}_{0 j} \\
\widehat{n}_{1 j} \\
\widehat{n}_{2 j} \\
0 \\
0
\end{array}\right) \frac{1}{2} \widehat{n}_{2 i}
$$

On the other hand, computation of integral coefficients $\varphi_{w}^{(u)}$ in $(55)$ can be summarized in the following expressions:

$$
\begin{aligned}
& \varphi_{w}^{(0)}=\varphi_{w}^{(0) A}-\varphi_{w}^{(0) B} \\
& \varphi_{w}^{(1)}=\varphi_{w}^{(1) A}-\varphi_{w}^{(1) B} \\
& \varphi_{w}^{(2)}=\varphi_{w}^{(1) A}+\varphi_{w}^{(1) B}-\lambda \mathcal{A} \varphi_{w}^{(1)}-\lambda \mathcal{B} \varphi_{w+1}^{(1)}
\end{aligned}
$$


being $\varphi_{w}^{(1) A}$ and $\varphi_{w}^{(1) B}$

$$
\begin{aligned}
& \left.\begin{array}{ll}
\varphi_{w}^{(0) A} & =\mathcal{I}\{w\}[a, b, c, d] \\
\varphi_{w}^{(1) A} & =\lambda \mathcal{J}\{w\}[a, b, c, d]
\end{array}\right\} \text { with }\left\{\begin{array}{l}
a=\left(\frac{1}{\lambda}-\mathcal{A}\right) \\
b=-\mathcal{B} \\
c^{2}=\mathcal{C}^{2}+a^{2} \\
d=\mathcal{D}-a \mathcal{B}
\end{array}\right. \\
& \left.\begin{array}{l}
\varphi_{w}^{(0) B}=\mathcal{I}\{w\}[a, b, c, d] \\
\varphi_{w}^{(1) B}=\lambda \mathcal{J}\{w\}[a, b, c, d]
\end{array}\right\} \text { with }\left\{\begin{array}{l}
a=-\left(\frac{1}{\lambda}+\mathcal{A}\right) \\
b=-\mathcal{B} \\
c^{2}=\mathcal{C}^{2}+a^{2} \\
d=\mathcal{D}-a \mathcal{B}
\end{array}\right.
\end{aligned}
$$

$\mathcal{I}\{m\}[a, b, c, d]$ and $\mathcal{J}\{m\}[a, b, c, d]$ are integrals that can be computed analytically [8] for different orders $0 \leq m \leq 4$. Resultant formulae will also depend on parameters $[a, b, c, d]$, which contain the information about the geometry of the two elements and their relative position. The complete development of these expressions and some discussions about their use can be found in a previous work [8].

Next, these analytical formulae are presented. In them, we have used geometrical parameters $[a, b, c, d]$ and the following relationships:

$$
\begin{gathered}
R^{2}=c^{2}-d^{2} ; \quad f=a-b d ; \omega^{2}=\left(c^{2}-a^{2}\right)\left(1-b^{2}\right)-(d-a b)^{2} \\
\nu^{2}=R^{2} b^{2}+f^{2} ; \quad r=\sqrt{(d+1)^{2}+R^{2}} ; \quad s=\sqrt{(d-1)^{2}+R^{2}} \\
\mathcal{I}\{n\}=\sum_{j=0}^{j=n}\left(\begin{array}{c}
n \\
j
\end{array}\right)(-d)^{j} \mathcal{I}_{1}\{n-j\} \\
\mathcal{I}_{1}\{n\}=\frac{(d+1)^{n+1}}{n+1} \ln (b(d+1)+r)-\frac{(d-1)^{n+1}}{n+1} \ln (b(d-1)+s) \\
\quad-\frac{b}{n+1} \mathcal{K}_{3}\{n+1\}, \quad \text { if } b^{2}=1 \\
\mathcal{I}_{1}\{n\}=\frac{(d+1)^{n+1}}{n+1} \ln (a+b+r)-\frac{(d-1)^{n+1}}{n+1} \ln (a-b+s) \\
+\frac{b f}{n+1} \mathcal{K}_{1}\{n+1\}-\frac{1-b^{2}}{n+1} \mathcal{K}_{1}\{n+2\} \\
-\frac{b R^{2}}{n+1} \mathcal{K}_{2}\{n+1\}+\frac{f}{n+1} \mathcal{K}_{2}\{n+2\}
\end{gathered}
$$




$$
\begin{aligned}
& \mathcal{J}\{n\}=\sum_{j=0}^{j=n}\left(\begin{array}{l}
n \\
j
\end{array}\right)(-d)^{j} \mathcal{J}_{1}\{n-j\} \\
& \mathcal{J}_{1}\{n\}=\mathcal{K}_{3}\{n+2\}+R^{2} \mathcal{K}_{3}\{n\} \\
& \mathcal{K}_{1}\{n\}=\frac{1}{1-b^{2}}\left[\frac{(d+1)^{n-1}-(d-1)^{n-1}}{n-1}\right. \\
& \left.+2 b f \mathcal{K}_{1}\{n-1\}-\left(R^{2}-f^{2}\right) \mathcal{K}_{1}\{n-2\}\right] \\
& \mathcal{K}_{1}\{1\}=\frac{1}{2\left(1-b^{2}\right)}\left[\ln \left(\frac{r^{2}-(a+b)^{2}}{s^{2}-(a-b)^{2}}\right)+2 b f \mathcal{K}_{1}\{0\}\right] \\
& \mathcal{K}_{1}\{0\}=\frac{1}{\omega} \arctan \frac{2 \omega}{c^{2}-a^{2}} \\
& \mathcal{K}_{3}\{n\}=\frac{(d+1)^{n-1}}{n} r-\frac{(d-1)^{n-1}}{n} s-\frac{(n-1) R^{2}}{n} \mathcal{K}_{3}\{n-2\} \\
& \mathcal{K}_{3}\{1\}=r-s \\
& \mathcal{K}_{3}\{0\}=\operatorname{ArgSh}\left(\frac{d+1}{R}\right)-\operatorname{ArgSh}\left(\frac{d-1}{R}\right) \\
& \mathcal{K}_{2}\{n\}=\frac{1}{1-b^{2}}\left[\mathcal{K}_{3}\{n-2\}+2 f b \mathcal{K}_{2}\{n-1\}-\left(R^{2}-f^{2}\right) \mathcal{K}_{2}\{n-2\}\right] \\
& \mathcal{K}_{2}\{1\}=\frac{1}{s}-\frac{1}{r}, \quad \text { if } b=f=0 \\
& \mathcal{K}_{2}\{0\}=\frac{1}{R^{2}}\left[\frac{d+1}{r}-\frac{d-1}{s}\right], \quad \text { if } \quad b=f=0 \\
& \mathcal{K}_{2}\{1\}=\frac{f}{\nu^{2}}\left[\operatorname{ArgTh}\left(\frac{a-b}{s}\right)-\operatorname{ArgTh}\left(\frac{a+b}{r}\right)\right] \\
& +\frac{R^{2} b}{\omega \nu^{2}}\left[\arctan \frac{\omega(a-b+b s)}{\omega^{2}+\left(d-1-a b+b^{2}\right)(d-1+s)}\right. \\
& \left.-\arctan \frac{\omega(a+b+b r)}{\omega^{2}+\left(d+1-a b-b^{2}\right)(d+1+r)}\right] \\
& \mathcal{K}_{2}\{0\}=\frac{b}{\nu^{2}}\left[\operatorname{Arg} \operatorname{Th}\left(\frac{a+b}{r}\right)-\operatorname{Arg} \operatorname{Th}\left(\frac{a-b}{s}\right)\right]
\end{aligned}
$$




$$
\begin{aligned}
+\frac{f}{\omega \nu^{2}} & {\left[\arctan \frac{\omega(a-b+b s)}{\omega^{2}+\left(d-1-a b+b^{2}\right)(d-1+s)}\right.} \\
& \left.-\arctan \frac{\omega(a+b+b r)}{\omega^{2}+\left(d+1-a b-b^{2}\right)(d+1+r)}\right]
\end{aligned}
$$

\section{References}

[1] ANSI/IEEE Std.80, IEEE Guide for safety in AC substation grounding (IEEE Publ., New York, 1986).

[2] J.G. Sverak, W.K. Dick, T.H. Dodds and R.H. Heppe, Safe substation grounding (Part I), IEEE Trans. on Power Apparatus and Systems 100 (1981) 4281-4290.

[3] R.J. Heppe, Computation of potential at surface above an energized grid or other electrode, allowing for non-uniform current distribution, IEEE Trans. on Power Apparatus and Systems 98 (1979) 1978-1988.

[4] D.L. Garrett and J.G. Pruitt, Problems encountered with the Average Potential Method of analyzing substation grounding systems, IEEE Trans. on Power Apparatus and Systems 104 (1985) 3586-3596.

[5] E. Durand, Électrostatique (Masson, Paris, 1966).

[6] L1. Moreno, Disseny assistit per ordinador de postes a terra en instal-lacions eléctriques (Tesina de Especialidad ETSECCPB, Universitat Politècnica de Catalunya, Barcelona, 1989).

[7] F. Navarrina, I. Colominas and M. Casteleiro, Analytical integration techniques for earthing grid computation by boundary element methods. Numerical Methods in Engineering and Applied Sciencies, Section VII: "Electromagnetics", 1197-1206 (H. Alder, J.C. Heinrich, S. Lavanchy, E. Oñate, B. Suárez (Editors); Centro Internacional de Métodos Numéricos en Ingeniería, CIMNE, Barcelona, 1992).

[8] I. Colominas, Cálculo y Diseño por Ordenador de Tomas de Tierra en Instalaciones Eléctricas: Una Formulación Numérica basada en el Método Integral de Elementos de Contorno (Ph.D. Thesis, E.T.S. de Ingenieros de Caminos, Canales y Puertos, Universidad de La Coruña, La Coruña, 1995).

[9] E.D. Sunde, Earth conduction effects in transmission systems (McMillan, New York, 1968).

[10] R. Dautray, J.L. Lions, Analyse mathématique et calcul numérique pour les sciences et les techniques, vol. 6 (Masson, Paris, 1988).

[11] M. Kurtovic, S. Vujevic, Potential of earthing grid in heterogeneous soil, International Journal for Numerical Methods in Engineering 31 (1991) 677688. 
[12] I. Stakgold, Green's functions and boundary value problems (Wiley, New York, 1979).

[13] O.D. Kellog, Foundations of potential theory, (Springer Verlag, Berlín, 1967).

[14] I. Colominas, F. Navarrina and M. Casteleiro, A validation of the boundary element method for grounding grid design and computation. Numerical Methods in Engineering and Applied Sciencies, Section VII: "Electromagnetics", 11871196 (H. Alder, J.C. Heinrich, S. Lavanchy, E. Oñate, B. Suárez (Editors); Centro Internacional de Métodos Numéricos en Ingeniería, CIMNE, Barcelona, 1992).

[15] C. Johnson, Numerical solution of partial differential equations by the finite element method (Cambridge Univ. Press, Cambridge, USA, 1987).

[16] T.J.R. Hughes, The finite element method (Prentice Hall, New Jersey, 1987).

[17] I. Colominas, F. Navarrina and M. Casteleiro, Fórmulas analíticas de integración para el cálculo de tomas de tierra mediante el método de los elementos de contorno. Métodos Numéricos en Ingeniería, Section: "Métodos Numéricos", 855-864 (F. Navarrina, M. Casteleiro (Editors); Sociedad Española de Métodos Numéricos en Ingeniería SEMNI, Barcelona, 1993).

[18] G. Pini and G. Gambolati, Is a simple diagonal scaling the best preconditioner for conjugate gradients on supercomputers?, Advances on Water Resources 13 (1990) $147-153$.

[19] M. Casteleiro, L.A. Hernández, I. Colominas and F. Navarrina, Memoria y Manual de Usuario del Sistema TOTBEM para Cálculo y Diseño Asistido por Ordenador de Tomas de Tierra de Instalaciones Eléctricas (E.T.S. de Ingenieros de Caminos, Canales y Puertos, Universidad de La Coruña, La Coruña, 1994).

[20] I. Colominas, J. Aneiros, F. Navarrina and M. Casteleiro, A Boundary Element Numerical Approach for Substation Grounding in a Two Layer Earth Structure. Advances in Computational Engineering Science, Section: "Recent Developments in Boundary Element Methods", 756-761 (S.N. Atluri, G. Yagawa (Editors); Tech Science Press, Atlanta, 1997). 